\title{
A Proposed Mathematical Model for Tripartite Synapse to Enhance Artificial Model for Artificial Neuron-Astrocyte Networks
}

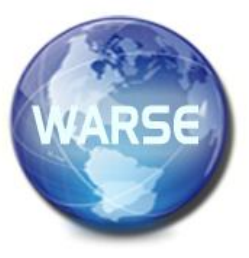

\author{
Bassam Abed $^{1}$, Belal Ayyoub ${ }^{2}$, Amelia Ritahani Ismail $^{3}$, Normaziah Abdul azizi ${ }^{4}$ Bassam Subaih \\ IIUM, Malaysia, bassamabed2000@gmail.com \\ BAU,Jordan,Belal_ayyoub@bau.edu.jo \\ IIUM,Malaysia, amelia@iium.edu.my \\ IIUM,Malaysianaa@iium.edu.my \\ BAU,Jordan, bassam_subaih@bau.edu.jo
}

[15] , [18]. Therefore, two concepts have been come forth, the Artificial Neuron-Glial Networks (ANGNs) and Spiking Neuron-Astrocyte Network (SNAN) to incorporate astrocyte in conventional ANN and SNN, respectively. The mechanisms underlying the molecular basis of tripartite synapse and the bidirectional interaction between astrocytes and neurons should be considered[4]. Firstly, when presynaptic neurons release neurotransmitter, e.g. Glutamate, spill to the synaptic cleft to communicate with other neurons, some of such glutamate could also spill out of the cleft and bind to receptors on the neighboring astrocyte. In turn, this stimulates the release of second messenger inositol 1,4,5-trisphospate (IP3) into the astrocytic cytoplasm, then IP3 opens channels to trigger calcium $\left(\mathrm{ca}^{+2}\right.$ ). When $\mathrm{ca}^{+2}$ levels increase above a threshold [20], the gliotransmitter (glutamate) is released from the astrocyte. In turn, the gliotransmitter glutamate can be released to synaptic cleft to modulate the synaptic activities by different mechanisms.However, in current study we propose mathematical models based on kinetic dynamics to mimic the communication between astrocytes-to-neurons (tripartite synapse). One of the pioneer researches in this context, a study by [1]whoimplemented different neuron-glia algorithms for multilayer ANGN to investigate the different astrocyte-neuron interactions by computational models for classification problem. Another study in context of learning algorithms to ANGN, [13] proposed a learning rule for ANGNs that fully automates the learning process based on coevolutionary Genetic Algorithms (GA) and can learn all parameters of the feed- forward, multilayer and fully connected ANGN without back propagation or lateral connections.In the current study, we propose mathematical modelbased on kinetic model for tripartite synapse to advance a new artificial model for artificial neuron-astrocyte networks

\section{Tripartite Synapse Model (TSM)}

The proposed model based on the two-state kinetic models to create a coherent neuron-astrocyte model in which subcellular, cellular, and network properties are described within the same formalism. As illustrated in Figure 1, which represents the proposed three pathways in tripartite synapse, 


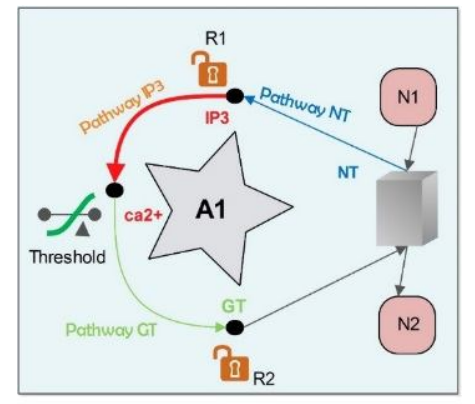

Figure 1:Proposed Pathways for Artificial Tripartite Synapse Model

In this simple model where $\mathrm{N} 1$ for presyanptic neurons and $\mathrm{N} 2$ postsynaptic neuron, the interaction between astrocyte A1 and the presynaptic neurons N1 and N2 has three pathways: pathway NT with blue dots arrow which represents the pathway between the neurontransmitter NT and the astrocytic second messanger IP3, pathway IP3 with red bold arrowwhich represents the pathway between IP3 and calcium $\mathrm{Ca}^{2+}$ and the pathway GT is represented by green arrow between $\mathrm{ca}^{2+}$ and GT. At the intersections of pathway IP3 and pathway GT, there are two channels R1 and $\mathrm{R} 2$, respectively. And at the intersection between pathway IP3 and calcium there is a threshold symbole to indicate that calcium has threshold to activate the pathway GT. We first consider that the relationship between the elements of the interaction: the neurotransmitter NT, the second messenger $I P_{3}$, the calcium $\mathrm{Ca}^{2+}$ and the gliotranmitter GT across the two channels $\left(R_{1}\right.$ and $\left.R_{2}\right)$ is stimulus-response relationship.

TSMdemonstrate how this relationship will elicit the channels considering that each channel has two states either close or open and the response of the channel changes its state depending on the stimulus. In the description of the model, there are three componants of tripartite synapse $\left(T_{1}\right)$ : an astrocyte $A$, population of presynaptic neurons $N_{1}$, and one postsynaptic neuron $N_{2}$. We propose to define the three pathways by the following terms: the pathway between neurotranmitter $(N T)$ and the second messenger $\left(I P_{3}\right)$ is termed $P_{N T-I P 3}$, the pathway between $I P_{3}$ and the calcium $\left(\mathrm{Ca}^{2+}\right)$ is called $P_{I P 3-c a}$ and the pathway between $c a^{2+}$ and gliotranmitter $(G T)$ is named $P_{c a-G T}$. The model has two channels: channel $1\left(R_{1}\right)$ between pathways $P_{N T-I P 3}$ and $P_{I P 3-c a}$, and the channel $2\left(R_{2}\right)$ between pathways $P_{I P 3-c a}$ and $P_{c a-G T}$.

However, to explain the model more clearly and in detail, we break the equations of the proposed astrocyte modelinto three steps based on the interactive pathways and they will be the focus of this section:

2.1 Neurotransmitter Pathway $\left(\boldsymbol{P}_{N T-I P 3}\right)$ : The initiation of $I P_{3}$ is dependent on the amount of the neurotransmitter released to astrocyte which considered as the quanta of total amount of the neurotransmitter released into the synaptic cleft. The synaptic efficacy has been typically defined as the distinctive feature of synapse determined by relevant factors such as the transmitter amount released to synaptic cleftor its simple definition as synaptic weight (single scalar, $w_{i j}$ ) to present the strength of the connection between neuron $j$ and neuron $i$. One of the most important subtleties is to quantify the synaptic efficacy. Hence, we propose to utilize any synaptic input equation to calculate the quanta of the neurotranmitter released to astrocyte by modifying the synaptic efficacy term, let assume the synaptic efficacy denoted as $T_{\text {syn }}$. Apparently, this proposed model of ATSM is not confined to $\mathrm{SNN}$, it can be applied to any ANN architecture by calculating the amount of neurotransmitter released into synapse through any term represents the synaptic strength or the efficacy. However, [7] proposed the relationship between the neurotransmitter concentration and the presynaptic voltage by kinetic models. Hence, we generalize this relationship to be applicable to astrocyte, and we propose the following equation to calculate the quanta of neurotransmitter concentration to stimulate astrocyte:

$$
N T=\frac{r_{N T^{*} T_{s y n}}}{1+\exp \left(-v(t)-v_{P} / k_{P}\right)}(1)
$$

Where $r_{N T}$ is the rate at which neurotransmitter interacts with astrocyte in order to control the quanta of the neurotransmitter, $v(t)$ is the presynaptic voltage, $k_{P}$ represent the steepness of the sigmoid function, and $v_{P}$ is the half activation voltage. Mainly, the effective rate of $I P_{3}$ production depends on the quanta of neurotransmitter $N T$ that is being released to astrocytes. The neurotransmitter NT governs the state across the respective channel, The firstorder kinetic scheme was introduced by [6]. The notation has been modified and simplified by [7]. We propose the kinetic model to represent the probability of the response $\left(I P_{3}\right)$ given the stimulus of neurotransmitter $(\mathrm{NT})$, can be written as:

$$
I P 3+N T \underset{\beta}{\stackrel{\alpha}{\rightleftharpoons} I P_{3}^{\prime}}
$$

When spike is ON because $N T>0$, The fraction of open gate IP3 for each time step $\Delta t$ is proposed as the following equation:

$$
I P 3=r+(I P 3-r) \exp \left(\frac{-\Delta t}{\tau_{r}}\right)
$$

Where $\Delta t$ is time step, $\tau_{r}$ and $\mathrm{r}$, are control variables where $\alpha, \beta, \beta^{\prime}$, and $T_{\text {max }}$ are constants. To calculate the change of the second messenger we propose the following equation:

$$
\frac{d I P 3}{d t}=\alpha[N T](1-I P 3)-\beta I P 3
$$

The variables $r$ and $\tau_{r}$ are defined as follows:

$$
r=\frac{\alpha T_{\max }}{\alpha T_{\max }+\beta}, \quad \tau_{r}=\frac{1}{\alpha T_{\max }+\beta^{\prime}}
$$

When spike is off and $S_{N T}=0$

$$
I P 3=I P 3 * \exp [-\beta(\Delta t)]
$$

$I P_{3}$ will be maintained whenever there is an input stimulus to the synapse. On the other hand of this spectrum, $I P_{3}$ depend on the stimulus frequency proportionally, i.e. the higher the input stimulus frequency, the higher the level of $I P_{3}[20]$. 
2.2 Second Messenger Pathway $\left(P_{I P 3-c a}\right)$ : The channel $R_{2}$ has the stimulus input of $I P_{3}$ (output of $R_{1}$ ) and the response output is calcium. The state diagram that represents the gating of calcium ion channel can be expressed as:

$$
C a 2+I P 3 \stackrel{\alpha}{\rightleftharpoons} \underset{\beta}{\rightleftharpoons} C a 2^{\prime}
$$

To compute the change of the calcium in which the increased $I P_{3}$ concentration triggers the calcium release from the ER and can, thus, evoke Calcium $\left(\mathrm{Ca}^{2+}\right)$ oscillations, hence, we propose the following equation:

$$
\frac{d[\mathrm{Ca} 2]}{d t}=\alpha[\mathrm{IP} 3](1-\mathrm{Ca} 2)-\beta \mathrm{Ca} 2
$$

The fraction of open gate ca2+ for each time step $\Delta t$ is proposed as the following equation:

$$
[c a 2]=s+([c a 2]-s) \exp \left(\frac{-\Delta t}{\tau c}\right)
$$

Where

$$
s=\frac{\alpha I P 3}{\alpha I P 3+\beta}
$$

2.3 Gliotransmitter Pathway $\left(P_{c a-G T}\right)$ : Increasing calcium concentration in the astrocyte cytoplasm triggers the production of astrocyte gliotransmitter (Glutamate) when $\mathrm{Ca}^{+2}$ crosses a threshold value $c a_{t h r}[20]$. We assume that gliotransmitter GT is some amount of calcium $\mathrm{Ca}^{2+}$ is defined as:

$$
\begin{gathered}
\text { GT }=\gamma *\left(\frac{1}{1+\exp [(-[c a 2]-\theta) / \sigma)]}\right), \text { if } \quad c a^{2+} \\
\geq c a_{t h r}, \\
0 \text { otherwise }
\end{gathered}
$$

Here, $\gamma$ is a control variable, $\theta$ is the value at which the function is half activated, $\sigma$ is the steepness. the calcium diffused from gap junction channels. Finally, to calculate the term $I_{\text {astro }}$, the astrocyte will release the gliotransmitter glutamate to the synapse as given in:

$$
I_{\text {astro }}(t)=\sigma \mathrm{GT}
$$

Here, $\sigma$ is a control parameter to control the strength of astrocyte.

\section{SIMULATIONS AND RESULTS}

The simulations have been performed for ATSM in MATLAB. We have used two neuron models, leaky integrate and fire (LIF) and Izhikevich model. Leaky Integrate and Fire (LIF) neuron model usually takes the form of the voltage when the current injection is constant over time as given:

$$
V(t)=E_{L}+R_{m} I_{e}+\left(V\left(t_{\circ}\right)-E_{L}-R_{m} I_{e}\right) \exp \left(-\frac{t-t_{\circ}}{\tau_{m}}\right)
$$

We simulate the following parameters values: membrane resistance $R_{m}=10 \mathrm{M} \Omega$, time constant $\tau_{m}=10 \mathrm{~ms}$, the current injection $I_{e}$ was $1.55, t_{0}$ is any reference time, $t$ is a single time-step $\Delta t=0.1 \mathrm{~ms}$. When the cell receives current injection, the membrane voltage increases with time until it reaches the AP threshold $V_{t h}=-55$, the voltage spikes and then immediately reset to its resting potential level $V_{\text {reset }}=-75 \mathrm{mV}$, where $V=E_{L}=-70 \mathrm{mV}$ at $t=$ 0 . We run our simulations for $1000 \mathrm{~ms}$ total (the initial current pulse of $I_{o}=0$ starts at time $t=0$ to $t_{\text {pulse }}=$ $200 \mathrm{~ms}$, the period of $600 \mathrm{~ms}$ with $I e=1.55$ and the last $200 \mathrm{~ms}$ with $I_{e}=0$ ), the firing rates between 1 and $100 \mathrm{~Hz}$. Firstly, we ran the LIF alone without astrocyte given the parameters above, the average firing rate was 25 (number of spikes per second) and the number of spikes fired was 16. Secondly, we run the LIF with the proposed TSM based on the assumption that the input of the injected current $\left(I_{e}=1.55\right)$ is equal the term $T_{s y n}$ in Equation 1and then we run our model with three pathways equations with the following parameters (fine tuning): $r_{N T}=0.1, t-t_{i}^{f} \Delta t=$ $0.1, \quad \tau_{1}=0.2, \quad S_{\infty}=N T, \quad S_{\circ}=0, \quad I_{\infty}=I P_{3}, \quad I_{\circ}=0.3$, $I P_{\text {thr }}=0.3, c a_{\text {thr }}=0.6, c a_{\text {external }}=0.2, \gamma=1, \sigma=1$.

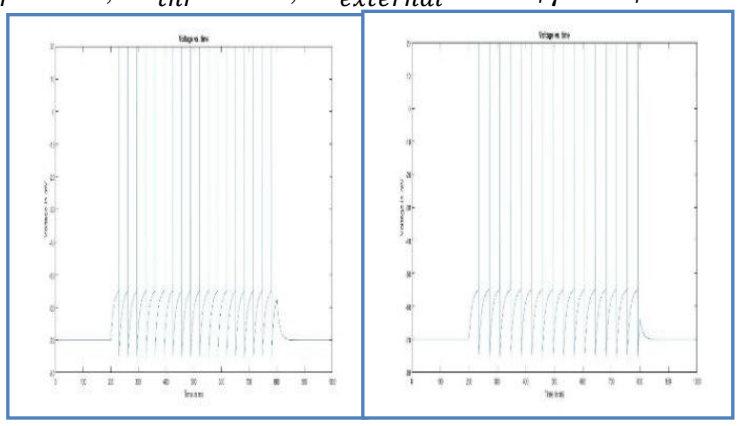

Figure 2: Number of spikes with two simulations: LIF with TSM (left) and without TSM (right)

The results showed that astrocyte has changed the average rate of firing to 30 and the number of the fired spikes to 18 as depicted in Error! Reference source not found.: LIF without TSM (right), LIF with TSM (left).

The second neuron model we have used in our simulation is Izhikevich model [11] , [12], which is based on the following two-dimensional system of ordinary differential equations of the form: $v^{\prime}=0.04 v^{2}+5 v+140-u+I$ and $u^{\prime}=a(b v-u)$, if $v \geq 30 \mathrm{mV}$, then $\left\{\begin{array}{c}v \leftarrow c \\ u \leftarrow u+d\end{array}\right.$.

The variable $v$ refers to the membrane potential and $u$ refers to the membrane recovery ( $v$ and $u$ will be reset when the spike reaches its highest value with $+30 \mathrm{mV}$ ), the variable $I$ refers to synaptic currents, the resting potential is between -70 and $-60 \mathrm{mV}$ depending on $b$, the threshold potential can be between $-55 \mathrm{mV}$ or and $-40 \mathrm{mV}$, the parameter $a$ represents the time scale of the recovery variable $u$ and the parameter $b$ represents the sensitivity of $u$ to the subthreshold fluctuations of $v$, the parameter $c$ represents the after-spike reset value of $v$, and the parameter $d$ represents after-spike reset of $u$. (the following parameters values were chosen (fine tuning): $a=0.02, b=0.2, c=$ $-65 \mathrm{mV}$, and $d=2$ ). Simulation has been done on sparse network of 10000 spiking neurons with 1000000 synaptic connections in real time (resolution $1 \mathrm{~ms}$ ). The synaptic 
connection weights between the neurons are given by the matrix $S=\left(s_{i j}\right)$, so that firing of the $j t h$ neuron instantaneously changes variable $v$ by $s_{i j}$ as shown in Figure 3 (left panel). Here we assumed that term $T_{\text {syn }}$ in Equation 3.1is equal to the matrix $S=\left(s_{i j}\right)$ term in Izhikevich model which is represented by a random number between 0 and 1 multiplied by 0.5 for excitatory neurons and by -1 for inhibitory neurons. The following parameters have been chosen for the TSM (fine tuning): $r_{N T}=0.1, t-t_{i}^{f}=$ $\Delta t=0.1, \tau_{1}=0.2, S_{\infty}=N T, S_{\circ}=0, I_{\infty}=I P_{3}, I_{\circ}=0.3$, $I P_{\text {thr }}=0.9, c a_{\text {thr }}=0.18, c a_{\text {external }}=0.2, \gamma=1, \sigma=1$.

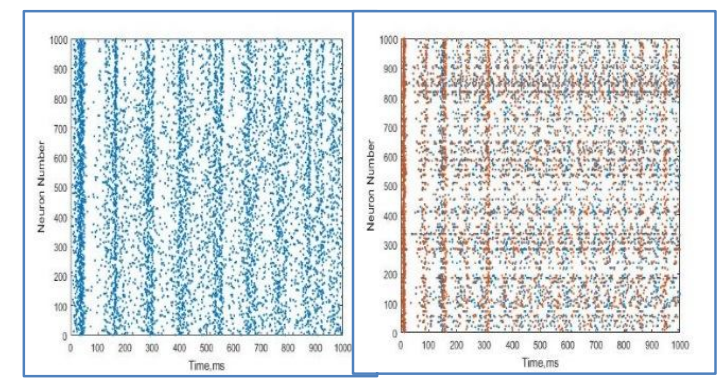

Figure 3: represents spiking activity (blue dots for Izhikevich model in left panel, red dots for TSM in right panel).

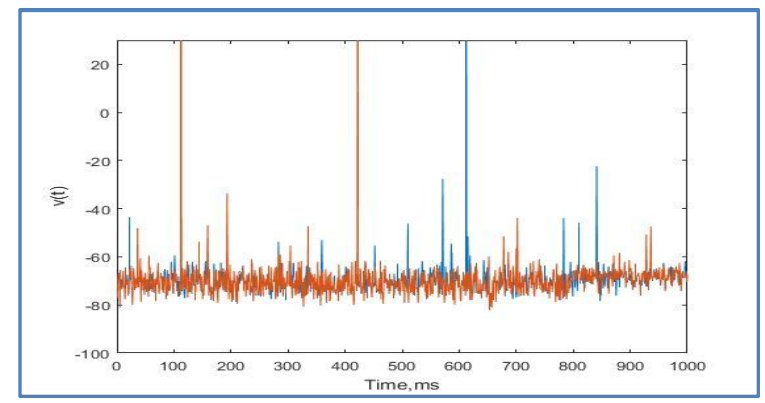

Figure 4:Spiking activity with Izhikevich model, red line for TSM

The simulation results showed that using of TSM has changed the spike behavior (rate and firing pattern) of Izhikevich model as shown in Figure 3 and Figure 4.

\section{DISCUSSION}

The simulations have been performed for TSM in two neuron models, leaky integrate and fire (LIF) as and Izhikevich model. The performance of the TSM was compared to standard LIF and Izhikevich model. We run our simulations for $1000 \mathrm{~ms}$ total and the results showed that using astrocyte (TSM) in the spiking neural network models such as LIF and Izhikevich has changed the average rate of firing (greater) and the number of the fired spikes (greater) or changed the spike behavior (rate and firing pattern) of the SNN models. Consequently, astrocytes increase the postsynaptic potential, in other words, astrocytes may help PSPs to reach the activation threshold to evoke the postsynaptic neuron to fire a spike and this indicates that the results of the simulation of real time astrocyte is matching the biological property related to the relationship between the amount of neuron stimuli (neurotransmitter) and the degree of astrocytic response: the greater the stimuli, the higher the level of functionality by astrocytes or the versatility of astrocytic functions.

Furthermore, the results matched the biological property related to the contribution of gliotransmission in the regulation of the release probability and their influence on the synaptic efficacy (weights) as illustration of the increase in the mean amplitude of excitatory postsynaptic activities. Moreover, the results matched the biological property which states that astrocytes represent an additional neuromodulatory system that acts in complement to the neuronal ones but with its own time and space domains. Finally, the results matched the biological properties which state that the astrocyte $\mathrm{Ca} 2+$ signal is not a stereotyped "on-off" response and can results in large, slow inward currents (SICs) able to significantly depolarize the cells and even to trigger their firing which has been proposed to induce their synchronous firing and enhance the frequency of spontaneous and evoked synaptic currents. In summary, the influence is typically changes in either (1) the frequencies of Excitatory Post Synaptic Potential (EPSP) or Inhibitory PSP (IPSP), (2) SICs, (3) rate of synaptic failure.

\section{CONCLUSION}

We proposed a model for the interaction between astrocyte and neurons in tripartite synapse to construct the tripartite synapse model (TSM) based on two- state kinetic model. We proposed three pathways to represent the interactions in tripartite synapse and label them as follows: firstly, the pathway from presynaptic neuron to astrocyte called neurotransmitter-second messenger pathway. Secondly, the pathway inside astrocyte at which the second messenger $I P_{3}$ elicits calcium $\mathrm{Ca}^{+2}$ named the second messenger-calcium pathway. Finally, the pathway at which calcium reaches the threshold and elicits the gliotransmitter to be released to synaptic cleft called calcium-gliotransmitter pathway. However, for the first two pathways, there are channels with gate probability to be opened subject to stimuli of the channel. For instance, second messenger $\left(I P_{3}\right)$ and calcium $\left(\mathrm{Ca}^{+2}\right)$ are two channels for the pathways: neurotransmittersecond messenger pathway and second messenger-calcium pathway, respectively. Whereas for the calciumgliotransmitter pathway, the probability of releasing gliotransmitter is subject to calcium threshold, if calcium crossed the threshold, amount of gliotransmitter will be released to synaptic cleft, presynaptic neuron, or postsynaptic neuron. Therefore, we proposed mathematical model for the tripartite synapse to mimic the channel gating mechanism based on the two-state kinetic model for neurotransmission proposed by [6] , [7]. Furthermore, we simulated the TSM with two neurons model: leaky integrate and fire (LIF) and Izhikevich model. We concluded that TSM model is biological inspired model by matching the results of the simulation with the biological properties presented in section 3. 2. Moreover, we concleded that TSM changed the behaviour of neuron models such as LIF and Izhikevich. 


\section{REFERENCES}

1. Alvarellos-González, A., Pazos, A., \& Porto-Pazos, A. B. (2012). Computational Models of Neuron-Astrocyte Interactions Lead to Improved Efficacy in the Performance of Neural Networks.Computational and Mathematical Methods in Medicine, 2012, 1-10.

2. Araque, A., Carmignoto, G., Haydon, P. G., Oliet, S. H. R., Robitaille, R., \& Volterra, A. (2014). Gliotransmitters travel in time and space. Neuron, 81(4), 728-739.

3. Araque, A., \& Navarrete, M. (2010). Glial cells in neuronal network function.Philosophical Transactions of the Royal Society of London. Series B, Biological Sciences, 365(1551), 2375-2381.

4. Araque, A., Parpura, V., Sanzgiri, R. P., \& Haydon, P. G. (1999). Tripartite synapses: Glia, the unacknowledged partner. Trends in Neurosciences.

5. Barker, A. J., \& Ullian, E. M. (2010). Astrocytes and synaptic plasticity.The Neuroscientist: A Review Journal Bringing Neurobiology, Neurology and Psychiatry, 16(1), 40-50.

6. Destexhe, a., Mainen, Z. F., \& Sejnowski, T. J. (1994). An Efficient Method for Computing Synaptic Conductances Based on a Kinetic Model of Receptor Binding. Neural Computation, 6(1), 14-18.

7. Destexhe, a, Mainen, Z. F., \& Sejnowski, T. J. (1998). Kinetic models of synaptic transmission: From Ions to Networks. Methods in Neural Modeling: From Ions to Networks, 1-25.

8. Fellin, T., \& Carmignoto, G. (2004). Neurone-toastrocyte signalling in the brain represents a distinct multifunctional unit.The Journal of Physiology, 559(Pt 1), 3-15.

9. Fields, R. D., \& Stevens-Graham, B. (2002). New insights into neuron-glia communication. Science (New York, N.Y.), 298(5593), 556-562.

10. Henneberger, C., Papouin, T., Oliet, S. H. R., \& Rusakov, D. A. (2010). Long-term potentiation depends on release of d-serine from astrocytes. Nature, 463(7278), 232-236.

11. Izhikevich, E. M. (2001). Resonate-and-fire neurons. Neural Networks, 14(6-7), 883-894.

12. Izhikevich, E. M. (2004). Which Model to Use for Cortical Spiking Neurons?IEEE Transactions on Neural Networks, 15(5), 1063-1070.

13. Mesejo, P., Ibáñez, O., Fernández-Blanco, E., Cedrón, F., Pazos, A., \& Porto-Pazos, A. B. (2015). Artificial Neuron-Glia Networks Learning Approach Based on Cooperative Coevolution. International Journal of Neural Systems, 25(04), 1550012.

14. Newman, E. A. (2003). New roles for astrocytes: Regulation of synaptic transmission. Trends in Neurosciences, 26(10), 536-542.

15. Parpura, V., Basarsky, T. A., Liu, F., Jeftinija, K., Jeftinija, S., \& Haydon, P. G. (1994). Glutamatemediated astrocyte-neuron signalling. Nature, 369(6483), 744-747.

16. Perea, G., \& Araque, A. (2009). Synaptic information processing by astrocytes. In Astrocytes in
(Patho)Physiology of the Nervous System (pp. 287-300). Springer US.

17. Porto-Pazos, A. B., Veiguela, N., Mesejo, P., Navarrete, M., Alvarellos, A., Ibáñez, O. Araque, A. (2011). Artificial Astrocytes Improve Neural Network Performance.PLoS ONE, 6(4), e19109.

18. Schummers, J., Yu, H., \& Sur, M. (2008). Tuned responses of astrocytes and their influence on hemodynamic signals in the visual cortex.Science (New York, N.Y.), 320(5883), 1638-1643.

19. Verkhratsky, A., Rodríguez, J. J., \& Parpura, V. (2012). Calcium signalling in astroglia. Molecular and Cellular Endocrinology, 353(1-2), 45-56.

20. Volman, V., Ben-Jacob, E., \& Levine, H. (2007a). The astrocyte as a gatekeeper of synaptic information transfer.Neural Computation, 19(2), 303-326.

21. Wade, J. J., McDaid, L. J., Harkin, J., Crunelli, V., \& Kelso, J. A. S. (2011). Bidirectional coupling between astrocytes and neurons mediates learning and dynamic coordination in the brain: A multiple modeling approach. PLOS ONE, 6(12), 1-24.

22. A.Alharthi, Convolutional Neural Network based on Transfer Learning for Medical Forms Classification 34, December 2019 International Journal of Advanced Trends in Computer Science and Engineering 8(6):34053411

23. V. Shilov, Modified Biperiodic Structures for Linear Particle Accelerators, December 2019, International Journal of Advanced Trends in Computer Science and Engineering 8(6):3535-3539 Follow journal, DOI: $10.30534 /$ ijatcse/2019/133862019 